\title{
GoWvis: a web application for Graph-of-Words-based text visualization and summarization
}

\author{
Antoine J.-P. Tixier, Konstantinos Skianis, and Michalis Vazirgiannis \\ Computer Science Laboratory \\ École Polytechnique, France
}

\begin{abstract}
We introduce GoWvis ${ }^{1}$, an interactive web application that represents any piece of text inputted by the user as a graph-ofwords and leverages graph degeneracy and community detection to generate an extractive summary (keyphrases and sentences) of the inputted text in an unsupervised fashion. The entire analysis can be fully customized via the tuning of many text preprocessing, graph building, and graph mining parameters. Our system is thus well suited to educational purposes, exploration and early research experiments. The new summarization strategy we propose also shows promise.
\end{abstract}

\section{Introduction}

The term independence assumption made by the traditional Bag-of-Words (BoW) representation of text comes with many limitations. One approach that challenges this assumption is the Graph-ofWords model (GoW). As shown in Figure 1, it represents a textual document as a graph whose vertices are unique terms in the document and whose edges capture term co-occurrence within a window of predetermined, fixed size, that is slided over the entire document from start to finish.

This approach is statistical, as terms are linked based on local context of co-occurrence only, regardless of any semantic or syntactic information (Distributional Hypothesis). Unlike BoW, GoW encodes term dependency and term order (via directed edges). The strength of the dependence between two words can also be captured by assigning a weight to the edge that links them. While other definitions can be used, we consider here edge

\footnotetext{
${ }^{1}$ https://safetyapp.shinyapps.io/ GoWvis /
}

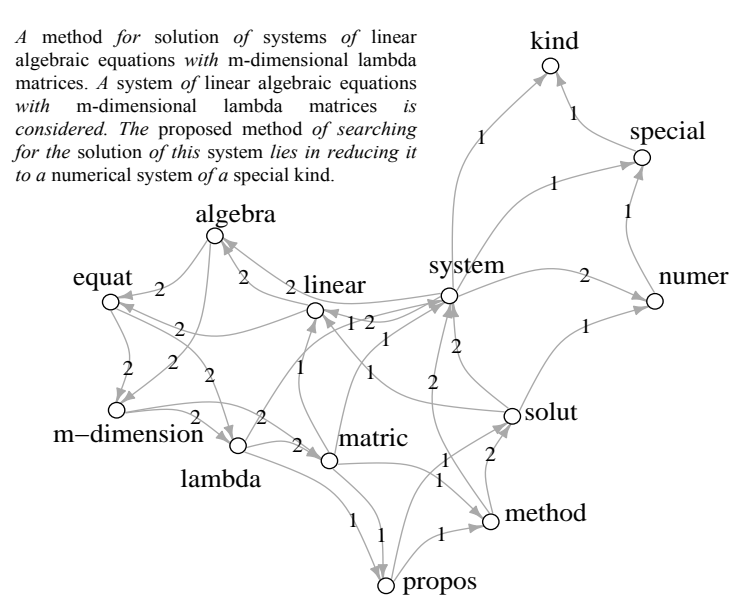

Figure 1: Graph-of-Words representation with POS-based screening, and directed, weighted edges. Non-(nouns and adjectives) in italic.

weights to be integers matching co-occurrence counts.

GoW can be tracked back to the works of (Mihalcea and Tarau, 2004) and (Erkan and Radev, 2004) who applied it to the tasks of unsupervised keyword extraction and extractive single document summarization. Notably, the former effort ranked nodes based on a modified version of the PageRank algorithm.

Recently, (Rousseau and Vazirgiannis, 2015) showed that degeneracy-based approaches (i.e., extracting dense, cohesive subgraphs) could outperform PageRank for unsupervised keyword extraction. We will show in subsection 3.4 how combining this strategy with graph clustering may improve summarization performance for multitopic documents. Other NLP tasks on which GoWbased approaches have reached new state-of-theart include ad-hoc information retrieval (Rousseau and Vazirgiannis, 2013) and document classification (Rousseau et al., 2015; Malliaros and Skianis, 2015). The high success, promising potential and visual nature of the GoW representation was the impetus for the development of GoWvis. 
The remainder of this paper is organized as follows: Section 2 provides some background on graph degeneracy and community detection, Section 3 presents our system, and finally, Section 4 concludes and discusses future work.

\section{Graph mining}

\subsection{Graph degeneracy}

k-core. A core of order $k$ (or $k$-core) of a graph $G$ is a maximal connected subgraph of $G$ in which every vertex $v$ has at least degree $k$ (Seidman, 1983). It is a relaxation of a clique: a $k$-core with $k+1$ members is a subgraph where every two nodes are adjacent, that is, a clique (Luce and Perry, 1949). In the classical unweighted case, edge weights are not taken into account and thus the degree of a node $v$ is simply equal to the number of its neighbors. In the weighted (or generalized) case, the degree of a vertex $v$ is the sum of the weights of its incident edges.

k-core decomposition. The $k$-core decomposition of a graph $G$ is the list of all its cores from 0 ( $G$ itself) to $k_{\max }$ (its main core). It forms a hierarchy of subgraphs that are recursively included in one another and whose cohesiveness and size respectively increases and decreases with $k$ (Seidman, 1983). A linear (resp. linearithmic) time algorithm for $k$-core decomposition can be found in (Batagelj and Zaveršnik, 2002) for the unweighted (resp. weighted) case. Both algorithms implement a pruning process that removes the lowest degree node at each step.

The core number of a node is the highest order of a core that contains this node. Nodes with high core numbers have the desirable property of not only being central (like nodes with high degree centrality) but also part of cohesive subgraphs with other central nodes (i.e., the other members of the upper cores). For this reason, they make, among other things, influential spreaders (Kitsak et al., 2010) and good keywords (Rousseau and Vazirgiannis, 2015)

The main core of a graph yields a fast (but rough) approximation of its densest subgraph. Indeed, it may contain in some cases a very large portion of the nodes of the graph. As (Seidman, 1983) puts it, $k$-cores should be regarded as seedbeds within which it is possible to find more cohesive subgraphs.

k-truss. A triangle-based extension of $k$-core that yields densest subgraphs is $k$-truss (Cohen,
2008). More precisely, the $k$-truss of a graph $G$ is the largest subgraph of $G$ in which every edge belongs to at least $k-2$ cycle subgraphs of length 3 (i.e., triangles). Put differently, every edge in the $k$-truss joins two vertices that have at least $k-2$ common neighbors.

k-truss decomposition. The $k$-truss decomposition of a graph $G$ is the set of all its $k$-trusses from $k-2$ to $k_{\max }$. The $k$-trusses correspond to densely connected subsets of the $k$-cores that can be viewed as their essential parts (Malliaros et al., 2016). The maximal $k$-truss thus yields a smaller and denser subgraph of $G$ that better approximates its densest subgraph. Nevertheless, the finer resolution of the $k$-truss decomposition comes at the cost of a greater complexity, polynomial in the number of edges (Wang and Cheng, 2012).

By analogy with $k$-core, the truss number of an edge is the highest order of a truss the edge belongs to. By extension, we define the truss number of a node as the maximum truss number of its incident edges, like in (Malliaros et al., 2016).

We wrote our own implementation of weighted $k$-core in $\mathrm{R}$ (R Core Team, 2015). For unweighted $k$-core, we used the igraph package (Csardi and Nepusz, 2006), and for $k$-truss, the $\mathrm{C}++$ implementation offered by (Wang and Cheng, 2012).

\subsection{Community detection}

While the $k$-core and $k$-truss decomposition algorithms converge towards the unique most cohesive subgraph of a graph, the task of community detection consists in clustering a graph into multiple groups within which connections are dense and between which they are sparse (Fortunato, 2010).

Many community detection algorithms have been proposed, of which some of the most popular are listed below. The fundamental Modularity function used by the first three algorithms measures the strength of the partition of a graph by comparing the number of within-group edges to the expected such number in a null model (Newman and Girvan, 2004).

The fast greedy algorithm (Clauset et al., 2004) merges at each step the pair of nodes that yields the largest gain in modularity until a single community remains. The best partition is the one associated with the greatest modularity value.

The multi-level (or Louvain) algorithm (Blondel et al., 2008) first aggregates each node with one of its neighbors such that the gain in modu- 
larity is maximized. Then, the groupings obtained at the first step are turned into nodes, yielding a new graph. This two-step process iterates until a peak in modularity is attained and no more change occurs.

The walktrap algorithm (Pons and Latapy, 2005) uses agglomerative hierarchical clustering with a random walk-based distance between vertices to obtain a set of subdivisions of the graph. The optimal clustering is given by the level of the hierarchy that maximizes modularity.

Finally, the infomap algorithm (Rosvall and Bergstrom, 2008) optimizes the map equation to find an optimal compression of a description of information flow in the graph. Unlike other aforementioned algorithms, infomap works for directed networks.

We used the $\mathrm{R}$ wrappers of the igraph $\mathrm{C}$ implementations of the algorithms presented above. Note that all igraph implementations can (optionally) take edge weights into account. Unless mentioned, all other parameters remained at their default values.

\section{GoWvis}

Our system was developed in R Shiny (Chang et al., 2015), and can be broken down into the four modules shown in Figure 2. The steps are sequential except the last two which are performed in parallel. In what follows, we present the tuning parameters involved at each step and discuss their individual impact (all other parameters being held constant).

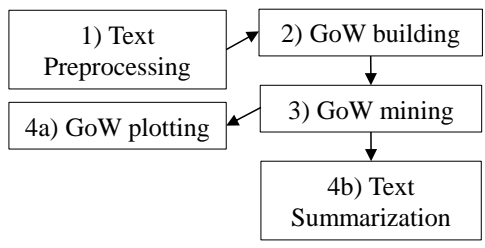

Figure 2: System architecture

\subsection{Text preprocessing}

The first module cleans the inputted text by (1) removing special characters, punctuation marks except the ones indicative of sentence boundary (used by the second module, see subsection 3.2) and intra-word dashes, (2) removing numbers except dates (like "2016"), and (3) tokenizing. In addition to R built-in functions, the stringr package (Wickham, 2015) is used here. Also, text is split into sentences using the implementation of the Apache OpenNLP Maxent sentence detector offered by the openNLP R package (Hornik, 2015). The list of sentences is eventually passed to the fourth module (see subsection 3.4). Additionally, the user is provided with the following tuning parameters:

Keep only nouns and adjectives? Boolean, defaults to TRUE. Uses openNLP's implementation of the Apache OpenNLP Maxent POS tagger to perform part-of-speech (POS) tagging. Then, following (Mihalcea and Tarau, 2004), only nouns and adjectives are kept.

Stopwords removal. Boolean, defaults to TRUE. Only actionable if no POS-based screening is performed. Removes common English stopwords $^{2}$ from the SMART information retrieval system.

Stemming. Boolean, defaults to TRUE. Retains only the stem of each term by implementing Porter's stemmer with the $\mathrm{R}$ snowballc package (Bouchet-Valat, 2014). For instance, when stemming is performed, win and winning are both collapsed to win. Stemming thus tends to yield smaller and denser graphs.

The unique words that passed the aforelisted preprocessing steps are then used as the nodes of the graph-of-words.

\subsection{Graph-of-Words building}

The graph-of-words is constructed by adding edges between the $n$ nodes previously obtained. Complexity is $O(n W)$ where $W$ is window size. The module offers the following tuning parameters:

Window size. Integer between 2 and 12, defaults to 3. Specifies the size of the window slided over the document. Values around 3 and 4 have been reported to work well (Mihalcea and Tarau, 2004; Malliaros and Skianis, 2015). Note that the larger the window, the denser the graph, since more edges are created while the number of nodes remains constant.

Build on processed text? Boolean, defaults to TRUE. Whether the window should be slided over the (1) processed or the (2) unprocessed text. May yield very different results, depending on the preprocessing steps that have been applied. Indeed,

\footnotetext{
${ }^{2}$ http://jmlr.org/papers/volume5/ lewis 04 a/al1-smart-stop-list/english. stop
} 
two words that are initially very distant in the original, unprocessed text and whose co-occurence would therefore not be captured may end up close to each other in the processed text if many words between them (e.g., stopwords) were removed as a result of preprocessing. Consequently, building the graph from the processed text tends to link more distant words and produce denser graphs than when using the unprocessed text.

Overspan sentences? Boolean, defaults to TRUE. If FALSE, an edge between two cooccurring words is only created (or if the edge already exists, its weight is only incremented) if the two words belong to the same sentence. The punctuation marks “.”, “,”, “!”, “?”, and “...” are used here as sentence boundaries.

Color. List, defaults to heat. A set of five built-in R palettes to color the nodes of the graph, including the color-blind-friendly gray.colors. Node colors match their core (or truss) number (also indicated in a legend) and go darker as $k$ increases.

\subsection{Graph-of-Words mining}

This module analyzes the graph-of-words returned at the previous step using graph degeneracy and community detection. The user can tweak the following parameters to customize the analysis:

Degeneracy. List, defaults to "weighted $k$ core". Choice of the graph decomposition method, among " $k$-core", "weighted $k$-core", and " $k$ truss". If "weighted $k$-core" is selected, the edge weights appear as edge labels in the plot.

Directed? Boolean, defaults to TRUE. Whether edge direction should be taken into account in computing node degree. Only actionable if a degree-based degeneracy algorithm has been selected (i.e., any but " $k$-truss"). When TRUE, edges in the plot feature arrows indicating their direction.

Mode. List, defaults to "all". Which of the incident edges of a node should be taken into account in computing its degree, between "all" (all edges), "in" (incoming edges only), or "out" (outgoing edges only). Only actionable if edge direction is taken into account, and only impacts the output of the $k$-core algorithms. Note that the default value "all" gives the same results as when edge direction is ignored, but generates a plot with arrow edges.

Community detection? List, defaults to "none".
Choice of the graph clustering algorithm, among "fast greedy", "louvain", "walktrap", "infomap", and "none". If not "none", each main community (see size threshold parameter below) is separately degenerated. If "walktrap", the user can select the length of the random walks between 2 and 8 (defaults to 4). If "infomap", the user can specify whether edge direction should be taken into account. Clustering increases coverage for multitopic documents.

Weighted? Boolean, defaults to FALSE. Whether edge weights should be used by the community detection algorithm. Only actionable if the community detection parameter is not "none". If TRUE, the edge weights appear as edge labels in the plot.

Size threshold. Numeric (from 0.4 to 1.0 , by 0.1 ), defaults to 0.8 . Only actionable if the community detection parameter is not "none". Percentile size threshold used to determine which communities should be considered to be main ones. For instance, the default value of 0.8 retains as main communities the ones whose sizes (i.e., number of nodes) exceed that of $80 \%$ of all detected communities. As will be further illustrated in subsection 3.4, this parameter enables the user to chose whether the summary should cover only the major or also the subtle topics of the document. Nonetheless, diminishing size threshold increases the risk of including irrelevant (or noise) topics in the summary.

\subsection{Text summarization}

The fourth module uses the results from the previous step (graph mining) to (1) extract keyphrases from and (2) select a subset of the original sentences in the document inputted by the user in an unsupervised manner. It is performed in parallel with the graph plotting module (see subsection 3.5).

1. Keyphrase extraction. The terms whose core (or truss) number is exactly equal to $k_{\max }-$ $p$ are used as seeds from which keyphrases $(n-$ grams) are reconstructed. $p$ is an integer parameter between 0 and 10 that lets the user navigate the core (or truss) hierarchy up and down. If $p=0$ (the default), the main core is used. Whenever $k_{\max } \leq p$, the user is informed that their selection is empty. In practice, one would want to retain all the words whose core (or truss) number is at least equal to $k_{\max }-p$, that is, the members 
of the $\left(k_{\max }-p\right)$-core (or truss), and this is indeed what we do for sentence selection (see "Sentence selection" paragraph below). Here though, we only use a single slice of the hierarchy (called a shell) to make it clear for the user how the process of keyword extraction and keyphrase reconstruction works.

Reconciliation is then performed by pasting together the seeds that are found adjacent in the original, unprocessed text. For example, if "algebra" and "linear" both belong to the selected shell and "linear algebra" is present in the text, the two seeds are collapsed and added to the set of candidate keyphrases. Duplicates and keyphrases included in higher order keyphrases are then discarded.

When community detection is used, as already explained, each main community is separately degenerated. The entire process of keyterm extraction and keyphrase reconstruction is then run for each main community, ensuring that keyphrases cover the main topics in the document.

Example. We created a two-topic 925-word document ${ }^{3}$ by drawing and intertwining an equal number of sentences from two Wikipedia articles, one about the website Stack Overflow (SO) and one about pizza. With all default parameters, the keyphrases extracted are all about SO: stack overflow, user, answer question... However, still with all default parameters, by simply enabling community detection (e.g., with "fast greedy"), the two topics are detected (answer question, pizza margherita, queen margherita).

Related work. Similarly, (Bougouin et al., 2013) have used clustering and graph mining for keyphrase extraction, but the other way around. They first group candidate keyphrases into topics via hierarchical clustering (with a word overlap distance), and then apply PageRank on a complete graph with topic nodes and edge weights based on keyphrase offset positions. Closer to our approach is that of (Grineva et al., 2009). Like us, they also observe that terms tend to cluster based on topic and that the largest communities correspond to the main themes in the document. However, they use a complete graph where edges are weighted based on Wikipedia-based semantic relatedness. Additionally, they select all the terms in the top-ranked communities whereas we extract only a highly cohesive subgraph from each main group.

\footnotetext{
${ }^{3}$ https://github.com/Tixierae/examples/
} blob/master/sopz.txt
2. Sentence selection. Unlike for keyphrase extraction, the entire $k_{\max }-p$ core (or truss) is used here as seedbed. Representative members are drawn from the list of sentences extracted from the original document (in subsection 3.1) following a three-step process: (1) sentences that do not contain any term belonging to the selected core (or truss) are pruned out, (2) the remaining sentences are ranked in decreasing order according to how many different central terms they feature, and finally, (3) sentences are selected one at a time from the top until a certain summary length has been reached. If two or more sentences have the same rank, the longest and least redundant is selected, where length is the number of words in the sentence and redundancy is computed in terms of word overlap with the current summary (stemming and stopword removal are performed based on user selection). The summary length tuning parameter is a decimal number (between 0.01 and 0.51 , by 0.05 , defaults to 0.01 ) indicating the percentage of total candidate sentences (from step 2 above) to include in the summary. Again, if community detection is performed, the process is run separately for each community, enabling coverage of the main topics in the document. In the previous example, using community detection generates a 11:1 compression ratio summary covering both themes (not shown here due to space limitations).

\subsection{Graph plotting}

Done in parallel with text summarization. Plots an interactive, dynamic browser-based representation of the graph-of-words using igraph and the visNetwork R package (Almende B.V. and Thieurmel, 2016).

\section{Conclusion and next steps}

We have presented GoWvis, a freely accessible web application providing an engaging illustration of the GoW concept and how it can be applied to unsupervised extractive single document summarization. Through trial and error, users can navigate the parameter space and develop an intuition as for which parameter values may be optimal for a given task and the particular type of text at hand. Future work should add support for directed degeneracy algorithms (Giatsidis et al., 2011). While showing promise, our summarization approach needs refinement and formal exper- 
iments to quantify how it compares to the stateof-the-art. When $p>0$, taking into account the core (or truss) numbers of terms could yield better sentence ranking.

\section{References}

Almende B.V. and Benoit Thieurmel, 2016. visNetwork: Network Visualization using 'vis.js' Library. $\mathrm{R}$ package version 0.2.1.

Vladimir Batagelj and Matjaž Zaveršnik. 2002. Generalized cores. arXiv preprint cs/0202039.

Vincent D Blondel, Jean-Loup Guillaume, Renaud Lambiotte, and Etienne Lefebvre. 2008. Fast unfolding of communities in large networks. Journal of statistical mechanics: theory and experiment, 2008(10):P10008.

Milan Bouchet-Valat, 2014. SnowballC: Snowball stemmers based on the C libstemmer UTF-8 library. $\mathrm{R}$ package version 0.5.1.

Adrien Bougouin, Florian Boudin, and Béatrice Daille. 2013. Topicrank: Graph-based topic ranking for keyphrase extraction. In International Joint Conference on Natural Language Processing (IJCNLP), pages 543-551.

Winston Chang, Joe Cheng, JJ Allaire, Yihui Xie, and Jonathan McPherson, 2015. shiny: Web Application Framework for $R$. R package version 0.12.2.

Aaron Clauset, Mark EJ Newman, and Cristopher Moore. 2004. Finding community structure in very large networks. Physical review E, 70(6):066111.

Jonathan Cohen. 2008. Trusses: Cohesive subgraphs for social network analysis. National Security Agency Technical Report, page 16.

Gabor Csardi and Tamas Nepusz. 2006. The igraph software package for complex network research. InterJournal, Complex Systems:1695.

Günes Erkan and Dragomir R Radev. 2004. Lexrank: Graph-based lexical centrality as salience in text summarization. Journal of Artificial Intelligence Research, pages 457-479.

Santo Fortunato. 2010. Community detection in graphs. Physics reports, 486(3):75-174.

Christos Giatsidis, Dimitrios M Thilikos, and Michalis Vazirgiannis. 2011. D-cores: Measuring collaboration of directed graphs based on degeneracy. In Data Mining (ICDM), 2011 IEEE 11th International Conference on, pages 201-210. IEEE.

Maria Grineva, Maxim Grinev, and Dmitry Lizorkin. 2009. Extracting key terms from noisy and multitheme documents. In Proceedings of the 18th international conference on World wide web, pages 661670. ACM

Kurt Hornik, 2015. openNLP: Apache OpenNLP Tools Interface. $\mathrm{R}$ package version $0.2-5$.

Maksim Kitsak, Lazaros K Gallos, Shlomo Havlin, Fredrik Liljeros, Lev Muchnik, H Eugene Stanley, and Hernán A Makse. 2010. Identification of influential spreaders in complex networks. Nature physics, 6(11):888-893.

R Duncan Luce and Albert D Perry. 1949. A method of matrix analysis of group structure. Psychometrika, 14(2):95-116.

Fragkiskos D Malliaros and Konstantinos Skianis. 2015. Graph-based term weighting for text categorization. In Proceedings of the 2015 IEEE/ACM International Conference on Advances in Social Networks Analysis and Mining 2015, pages 1473-1479. ACM.

Fragkiskos D Malliaros, Maria-Evgenia G Rossi, and Michalis Vazirgiannis. 2016. Locating influential nodes in complex networks. Scientific reports, 6:19307.

Rada Mihalcea and Paul Tarau. 2004. Textrank: Bringing order into texts. Association for Computational Linguistics.

Mark EJ Newman and Michelle Girvan. 2004. Finding and evaluating community structure in networks. Physical review E, 69(2):026113.

Pascal Pons and Matthieu Latapy. 2005. Computing communities in large networks using random walks. In Computer and Information Sciences-ISCIS 2005, pages 284-293. Springer.

R Core Team, 2015. R: A Language and Environment for Statistical Computing. R Foundation for Statistical Computing, Vienna, Austria.

Martin Rosvall and Carl T Bergstrom. 2008. Maps of random walks on complex networks reveal community structure. Proceedings of the National Academy of Sciences, 105(4):1118-1123.

François Rousseau and Michalis Vazirgiannis. 2013. Graph-of-word and tw-idf: new approach to ad hoc ir. In Proceedings of the 22nd ACM international conference on Conference on information \& knowledge management, pages 59-68. ACM.

François Rousseau and Michalis Vazirgiannis. 2015. Main core retention on graph-of-words for singledocument keyword extraction. In Advances in Information Retrieval, pages 382-393. Springer.

François Rousseau, Emmanouil Kiagias, and Michalis Vazirgiannis. 2015. Text categorization as a graph classification problem. In $A C L$, volume 15, page 107.

Stephen B Seidman. 1983. Network structure and minimum degree. Social networks, 5(3):269-287.

Jia Wang and James Cheng. 2012. Truss decomposition in massive networks. Proceedings of the VLDB Endowment, 5(9):812-823.

Hadley Wickham, 2015. stringr: Simple, Consistent Wrappers for Common String Operations. R package version 1.0.0. 